\section{Research Square}

Preprints are preliminary reports that have not undergone peer review.

They should not be considered conclusive, used to inform clinical practice, or referenced by the media as validated information.

\title{
Impact of Physical Activity Intensity on Longitudinal Trajectories of Cognitive Function and Depressive Symptoms in Middle-aged and Older Chinese Adults: A Longitudinal Study
}

\author{
Wen Zhang \\ University of Hong Kong \\ Tingxuan Wang \\ University of Hong Kong \\ Anni Wang ( $\square$ wanganni1990@163.com) \\ Fudan University
}

\section{Research Article}

Keywords:

Posted Date: March 7th, 2022

DOI: https://doi.org/10.21203/rs.3.rs-1398716/v1

License: (c) This work is licensed under a Creative Commons Attribution 4.0 International License. Read Full License 


\section{Abstract Background}

Cognitive decline and depressive symptoms are very common in the ageing process. Physical activity (PA) has been demonstrated to be associated with the improvement of cognitive function and the reduction of depressive symptoms. However, little is known about how longitudinal trajectories of cognitive function and depressive symptoms are impacted by varying levels of PA intensity. In this study, we aimed to test whether and to what degree the rate of change in longitudinal trajectories of cognitive function and depressive symptoms over time was associated with PA intensity

\section{Method}

The data were from the China Health and Retirement Longitudinal Study, a nationally representative household study of the Chinese population aged 45 years and older. The sample included 5,025 middle-aged and older adults (mean age $=57$ ) who self-reported their cognitive function, depressive symptoms, and PA intensity (vigorous PA, moderate PA, and light PA) every two years from 2011 to 2018.

\section{Result}

On average, every 2 years, cognitive function decreased by 0.09 points ( $95 \% \mathrm{Cl}$ : $[-0.15,-0.03], P<0.01)$, while depressive symptoms increased by 0.22 points (95\% Cl: $[0.14,0.30], \mathrm{P}<0.001)$. Middle-aged and older adults with more frequent moderate PA were more likely to report better cognitive function $(\beta=0.02,95 \% \mathrm{Cl}:[0.002,0.04])$, while those with more frequent light PA were more likely to have fewer depressive symptoms ( $\beta=-0.03,95 \% \mathrm{Cl}:[-0.06,0.01])$. Rates of reduction in cognitive function were lower among middle-aged and older adults with more moderate PA. There was no reduction in cognitive function when the participants reported a minimum of 6 days of moderate PA per week.

\section{Conclusion}

Moderate PA could slow the reduction in cognitive function and light PA could slow the progression of depressive symptoms. A targeted intensity of PA may be more useful in obtaining the maximal improvement in cognitive function and mental health among middle-aged and older people. At least 6 days of moderate PA per week is advised to prevent cognitive impairment.

\section{Introduction}

With increasing life expectancy, most countries become ageing societies. The ageing population has shown consistent trends, arising first in developed countries and then almost all developing countries [1]. According to the United Nations (UN), there were more than 960 million adults aged over 60 years old in the global population in 2017 [2]. The WHO predicted that the proportion of elderly individuals worldwide will double from $12 \%$ in 2015 to $22 \%$ in 2050 and that nearly $80 \%$ of this population will live in low- and middle-income countries by 2050 [3]. The ageing population has become a global public health issue, and it brings heavy financial burdens to society. Developing countries undergoing this demographic shift face more challenges than developed countries in promoting health and social system sustainability [4]

Late-life depression and a decline in cognitive function are frequent outcomes and significant reasons for illness or even death among the ageing elderly individuals [5]. Ageing has noticeable consequences on elderly individuals' daily activity, independent living, social functioning, and physical and mental health problems [6]. In China, existing studies have demonstrated a rising prevalence of cognitive diseases such as dementia among middle-aged and older adults, ranging from $0.75-11.86 \%$ between 1985 and 2013 [7, 8]. Data have shown that depressive symptoms are one of the impactful accompanying symptoms in older adults with cognitive decline [9]. Notably, several critical cognitive functions (e.g., episodic memory) deteriorate with ageing, even among middle-aged adults [10]. It is critical to enhance the prevention of cognitive decline and mental health conditions for healthy middleaged and elderly individuals and to propose a practical approach to delaying cognitive impairment among this population.

According to a growing body of evidence, physical activity (PA) improves cognitive function and mental health. A systematic review of 27 identified studies examined the impact of physical exercise and reported a significant improvement in cognitive function; however, the review failed to provide a comprehensive assessment of different dimensions of PA (e.g., types, intensity) on cognitive performance [11]. Similarly, another study targeting young and middle-aged adults also measured this association, reporting that only a higher level of PA increased executive functioning [8]. Studies focusing on the impact of PA on mental status also revealed that a higher level of PA led to lower odds of life-long depression and anxiety symptoms [12, 13]. However, the inconsistent results regarding different intensities and frequencies of PA cannot be integrated for a more representative population, and most observational studies fail to predict causality. There is a paucity of long-term evidence investigating changes and associations in the Chinese population.

Recent ageing-related studies have reported a growing demand to support the elderly population in the long term. Studies examining risk factors for cognitive function and mental health issues should be conducted from a more accessible and daily life perspective. Based on the above research gap, this study aimed to assess the trajectory of cognitive function and depressive symptoms by using longitudinal data and further explore whether different 
levels of PA intensity could predict cognitive function and depressive symptoms among general middle-aged and older Chinese adults. The findings on interventions that strengthen the prevention of cognitive decline and depression have significance for public health.

\section{Methods}

\section{Sample and data}

Data were from an ongoing longitudinal study of the China Health and Retirement Longitudinal Study (CHARLS). The CHARLS collected a national representative cohort of Chinese residents aged 45 and older to gather a wide range of personal health information and social and economic data for geriatric and health policy research. Approximately 150 areas (counties) within 28 provinces in China were first selected proportional to population size, and 3 villages/communities were then chosen from each county as primary sample units (PSUs). Eighty households were chosen at random from each of the 450 PSUs, with 24 being investigated. If there were persons 45 years or older in the household, one of them was chosen as a respondent at random, and both the respondents and their spouses were assessed using structured questionnaires. The data currently include 4 waves, including baseline (wave 1: 2011) and follow-ups every 2 years at wave 2 (2013), wave 3 (2015), and wave 4 (2018). Eventually, a total of 17,708 participants from 10,257 households were recruited at baseline. A detailed description of the cohort information and methods has been reported elsewhere [14].

Of 17,708 participants at baseline, 12,683 participants were excluded because of (1) age less than 45 years old; (2) lack of data on PA intensity; (3) diagnosis of Parkinson's disease, Alzheimer's disease, brain atrophy, or stroke attachment; and (4) lack of cognitive and depressive symptom assessment data. Eventually, a total of 5,025 participants were eligible for the current analysis. A total of $3,617,3,606$, and 2,672 of the respondents provided cognitive function data during follow-up in wave 2 , wave 3 , and wave 4, respectively, while 4,198, 4,125, and 3,764 provided depressive symptom data, respectively. The flow chart of the current study is shown in Figure 1.

\section{Measures}

\section{Physical activity intensity}

PA intensity was categorized according to three levels, including vigorous PA, moderate PA, and light PA [15]. Vigorous PA was defined as activities that made individuals breathe much harder than normal, including digging, aerobics, heavy lifting, ploughing, quick cycling, and cycling with a heavy load. Moderate PA was defined as activities that made individuals breathe faster than normal, including cycling at a normal speed, carrying light items, practicing tai-chi, and fast walking. Light PA referred to walking for leisure or recreation, walking at work, and travelling between places. The respondents were asked whether they performed vigorous PA, moderate PA, or light PA for at least 10 minutes every week and how many days a week they took part in the corresponding type of PA. In this study, the frequency of vigorous PA, moderate PA, and light PA with a range of 0-7 days/week was included in the analyses.

\section{Cognitive function}

Following a previous study [16], cognitive function was investigated via the Telephone Interview of Cognitive Status (TICS, measuring mental intactness), with a score range of 0-9; a word recall test (assessing episodic memory), with a score range of 0-20; and a figure drawing test (assessing visuospatial abilities), with a score range of 0-1. The TICS included a serial $7 \mathrm{~s}$ test (range $0-5$ ) and date naming (range $0-4$ ). In the serial $7 \mathrm{~s}$ test, the participants were asked to subtract 7 from 100 for up to five consecutive times. In the date naming test, the participants were asked to correctly report the date (day, month, year) and day of the week. In the word recall test, the participants were asked to recall 10 different words both immediately and $4-10$ min later. In the figure drawing test, the participants were asked to draw overlapping pentagons. Cognitive function was assessed based on the sum score of these tests, and the total score ranged from 0 to 30 . A higher total score indicated better cognitive function.

\section{Depressive symptoms}

Depressive symptoms were assessed by the Chinese version of the 10-item Epidemiologic Studies Depression Scale (CESD), which has been validated and widely used in Chinese older adults [17]. The participants were asked, "How often did you feel this last week?" They were asked to rate this question on a 4-point scale from 1 (rarely or one) to 4 (most or all of the time, 5-7 days). The total score ranged from 0 to 40 , and a higher score suggested a higher level of depressive symptoms.

\section{Covariates}

The covariates included baseline age, sex, educational attainment, marital status, current residence location, number of chronic diseases, and activities of daily living (ADLs). Educational attainment was categorised as "no formal education", "elementary school and middle school", and "high school and higher". Marital status was grouped as "married/partnered" and "unmarried". The current resident locations included "rural" and "urban". The number of chronic diseases was used to assess self-reported chronic diseases that had been diagnosed (such as diabetes, cancer, heart attack, chronic lung diseases, and liver disease). ADLs were assessed to determine if the participants had difficulties in bathing, eating, dressing, getting in and out of bed, using the toilet, and controlling urine, with a score range of 0-6.

\section{Statistical analysis}


The demographic characteristics included the mean (standard deviation, SD), median (interquartile range, IQR), and number (percentage). To study the impact of PA intensity on trajectories of cognitive functioning and depressive symptoms across the four waves, multilevel mixed-effects generalised linear models (GLMs) with a Gaussian distribution and an identity link function were used. The repeated measurements of data nested within individuals were taken into consideration by these multilevel models. To determine if trajectories differed by level of different PA intensity (vigorous PA, moderate PA, and light PA), we employed interaction terms (i.e., vigorous PA * wave, moderate * wave, and light PA * wave). If a significant effect of the interaction term was to be determined, we then tested marginal effects and plotted the trajectories of cognitive functioning and depressive symptoms by this interaction term. Covariates reported at baseline were adjusted in all models. In the sensitivity analysis, we performed multiple imputations by chained equations (MICE) to handle missing data [18]. A total of 10 imputed data sets were obtained, and the results were pooled by Rubin's rule. Similar results were found (Supplementary Table S1). Analyses were performed using R version 4.0.4.

\section{Results}

\section{Characteristics of the participants}

The characteristics of the participants are shown in Table 1. The current study was based on 5,025 participants (mean age $=57$, and 2,545 (49.4\%) were female). Most (85.3\%) had less than a lower high school education, and over half (56.9\%) lived in rural areas. During the 7-year follow-up, there was a reduction in moderate PA but no change in vigorous PA or light PA.

\section{The trajectories of cognitive function and depressive symptoms}

In the multilevel mixed-effects GLM, we identified a decreasing trend in the trajectories of cognitive function and an increasing trend in depressive symptoms after adjusting for covariates. On average, every 2 years, cognitive function decreased by 0.09 points $(95 \% \mathrm{Cl}$ : [-0.15, -0.03$], P<0.01)$, while depressive symptoms increased by 0.22 points $(95 \% \mathrm{Cl}$ : $[0.14,0.30], \mathrm{P}<0.001)$ (Supplementary Table S2). Compared to the mean score at baseline in 2011 , cognitive function decreased by $0.6 \%$ (95\% Cl:[1\%, $0.2 \%]$, while depressive symptoms increased by $2.8 \%$ (95\% Cl:[2\%,4\%]).

\section{The impact of PA intensity on the trajectories of cognitive function and depressive symptoms}

The trajectories of cognitive function and depressive symptoms were also altered by levels of different levels of PA (Table 2 and Figure 2 ). Middle-aged and older adults with more frequent moderate PA were more likely to report better cognitive function $(\beta=0.02,95 \% \mathrm{Cl}$ [0.002,0.04]), while those with more frequent light PA were more likely to indicate lower depressive symptoms ( $\beta=-0.03,95 \% \mathrm{Cl}:[-0.06,0.01])$. Rates of cognitive function reduction were lower among middle-aged and older adults with more moderate PA. There was no reduction in cognitive function when the participants reported a minimum of 6 days of moderate PA in a week. The increase in depressive symptoms was slower among middle-aged and older adults with more frequent light PA.

\section{Discussion}

To the best of our knowledge, this is the first study to identify how different levels of PA intensity predict and improve the trajectories of cognitive function and depressive symptoms in Chinese middle-aged and older adults. In this study, we analysed a nationally representative sample of Chinese middle-aged and older adults from 2011 to 2018 to examine the trajectories of cognitive function and depressive symptoms. Consistent with previous studies in other countries, we found that cognitive function deteriorated and that depressive symptoms worsened over time among Chinese middle-aged and older adults $[19,20]$. Reduction in cognitive function was shown to be slowed in middle-aged and older adults with more frequent moderate PA, while the progression of depressive symptoms was demonstrated to be delayed in those with more frequent light PA. These findings were robust to sensitivity analyses by conducting models with imputed missing data.

Middle-aged and older adults with more frequent moderate PA had delayed declines in cognitive function, and there was no reduction when they reported a minimum of 6 days of moderate PA in a week. This finding adds to the existing knowledge about the negative association between PA and cognitive function reduction. In this study, the impact of moderate PA instead of vigorous or light PA on cognitive function was highlighted. This finding suggests a potential inverted U-shaped dose-response between PA and cognitive function. A previous study suggested that high-intensity PA could boost the cardiovascular system, which leads to an increase in cerebral blood flow and oxygen delivery to the brain and then impacts cognitive performance [21]. On the other hand, vigorous PA necessitates more activation of the premotor cortex and supplementary motor regions at the expense of the prefrontal cortex, leading to the prefrontal cortex's higher-order processes being disengaged [22]. Moderate PA might improve numerous domains of cognition by activating the arousal mechanism of the reticulum system [22]. We also found that more frequent light PA, but not moderate and vigorous PA, slowed increases in depressive symptoms. Light PA referred to walking for leisure or recreation, walking at work, and travelling between places in this study. These types of PA might lead to more social interaction with other people and social participation, which might alleviate depressive symptoms [23]. However, this finding was not consistent across all age groups, although similar results have also been reported in older US adults [24]. Kandola's study found that moderate-to-vigorous PA at the age of 12 was associated with depressive symptoms at the age of 18 , while the link was also found in light PA [25]. There might be a need for stronger evidence, i.e., a meta-analysis to examine the different levels of PA intensity on different age groups in depressive symptom reduction in future research.

\section{Implications}


According to the newest guideline on PA from the World Health Organization [26], at least 150-300 minutes of moderate-intensity PA or at least 75-150 minutes of vigorous PA throughout the week is suggested. However, in this study, compared with vigorous PA, moderate PA was suggested to be a more effective way to prevent a reduction in cognitive function among middle-aged and older adults. The study also indicated that it would also be beneficial for middle-aged and older adults to engage in more frequent light PA to reduce depressive symptoms. Future guidelines should reconsider the benefits of vigorous PA in middle-aged and older adults and incorporate light PA if more evidence corroborates the present findings. Encouraging older adults to engage in light PA might be more realistic since the ageing process, along with underlying chronic conditions, may restrict their capacity and motivation to engage in vigorous PA. Furthermore, a minimum of 6 days of moderate PA per week (>10 mins per day) was recommended for older adults since no reduction in cognitive function was predicted among those with a minimum of 6 days of moderate PA in a week in this study.

\section{Limitations and strengths}

This study had several limitations. First, as with all self-reported assessments, the participants may have underreported challenges during investigations because they were unconscious of or unwilling to admit the presence of mental health issues. Second, in our study, depressive symptoms were assessed based only on feelings in the previous week, which might alter over a year. Third, we were unable to account for unmeasured variables that may be crucial to include in our models, such as healthcare access, because of the nature of secondary data analysis. Despite these existing limitations, there were some strengths in this study. A nationally representative data collection of Chinese middle-aged and older adults was employed, generating more credible results. We also identified the marginal effect of different types of PA intensity on cognitive function and depressive symptoms, providing more detailed recommendations for future intervention studies or guidelines. A sensitivity analysis was conducted to ensure the robustness of our findings, which revealed the same trajectory patterns.

\section{Conclusion}

Depressive symptoms frequently coexist with cognitive impairment during the ageing process, regardless of the cultural context, which is related to a higher disease burden. In this study, we found that moderate PA and light PA rather than vigorous PA could slow the reduction in cognitive function and the progression of depressive symptoms. In middle-aged and older adults, a tailored intensity of PA may be more beneficial in achieving the maximum improvement in cognitive function and mental health. At least 6 days of moderate PA per week (> 10 minutes per day) is strongly recommended to prevent cognitive impairment since no cognitive decline was predicted in those with at least 6 days of moderate PA per week.

\section{Abbreviations}

ADLs Activities of Daily Living

CESD Chinese version of the 10-item Epidemiologic Studies Depression Scale

CHARLS China Health and Retirement Longitudinal Study

Cl Confidence Interval

GLMs Generalized linear models

MICE Multiple imputations by chained equations

PA physical activity

PSU Primary sample units

SD standard deviation

UN United Nations

WHO World Health Organization

\section{Declarations}

\section{Ethics approval and consent to participate}

The data were from the China Health and Retirement Longitudinal Study, which was approved by the Ethical Review Committee of Beijing University (IRB00001050-11015) and signed informed consent was obtained from all participants. All methods were carried out in accordance with relevant guidelines and regulations.

\section{Consent for publication}

Not applicable. 
Availability of data and materials

The datasets used in the current study are accessed in the CHARLS website (http://forum.charls.pku.edu.cn/) after the users apply in their website.

\section{Competing interests}

The authors declare that they have no competing interests.

\section{Funding}

There is no fund for this study.

\section{Authors' contributions}

ZW and AW conceived of the presented idea. ZW conducted the data analyses. ZW and TW wrote the main manuscript. All authors reviewed the manuscript.

\section{Acknowledgements}

We would like to thank all the participants and staffs in the China Health and Retirement Longitudinal Study (CHARLS).

\section{References}

1. World Health Organization. Global health and ageing 2021a [cited 202224 January]. Available from: chromeextension://efaidnbmnnnibpcajpcglclefindmkaj/viewer.html?pdfurl = https\%3A\%2F\%2Fwww.who.int\%2Fageing\%2Fpublications\%2Fglobal_health.pdf\&clen = 1662875\&chunk = true.

2. United Nations. World population ageing 2017 [cited 202223 January]. Available from: chromeextension://efaidnbmnnnibpcajpcglclefindmkaj/viewer.html?pdfurl = https\%3A\%2F\%2Fwww.un.org\%2Fen\%2Fdevelopment\%2Fdesa\%2Fpopulation\%2Fpublications\%2Fpdf\%2Fageing\%2FWPA2017_Highlights.pdf\&clen $=11211395 \&$ chunk $=$ true .

3. World Health Organization. Ageing and health 2021b [cited 202224 January]. Available from: https://www.who.int/news-room/factsheets/detail/ageing-and-health.

4. World Health Organization. Ageing 2021c [cited 202224 January]. Available from: https://www.who.int/health-topics/ageing\#tab=tab_1.

5. Wei J, Ying M, Xie L, Chandrasekar EK, Lu H, Wang T, et al. Late-life depression and cognitive function among older adults in the US: The National Health and Nutrition Examination Survey, 2011-2014. Journal of psychiatric research. 2019;111:30-5.

6. Wu Z, Zhang H, Miao X, Li H, Pan H, Zhou D, et al. High-intensity physical activity is not associated with better cognition in the elder: evidence from the China Health and Retirement Longitudinal Study. Alzheimer's Research \& Therapy. 2021;13(1):182. doi: 10.1186/s13195-021-00923-3.

7. Zhu Y, Liu H, Lu X-L, Zhang B, Weng W, Yang J, et al. Prevalence of dementia in the People's Republic of China from 1985 to 2015: a systematic review and meta-regression analysis. BMC Public Health. 2019;19(1):578. doi: 10.1186/s12889-019-6840-z.

8. Cox EP, O'Dwyer N, Cook R, Vetter M, Cheng HL, Rooney K, et al. Relationship between physical activity and cognitive function in apparently healthy young to middle-aged adults: A systematic review. J Sci Med Sport. 2016;19(8):616-28. Epub 2015/11/11. doi: 10.1016/j.jsams.2015.09.003. PubMed PMID: 26552574.

9. Zou C, Chen S, Shen J, Zheng X, Wang L, Guan L, et al. Prevalence and associated factors of depressive symptoms among elderly inpatients of a Chinese tertiary hospital. Clinical interventions in aging. 2018;13:1755.

10. Cohen JA, Verghese J, Zwerling JL. Cognition and gait in older people. Maturitas. 2016;93:73-7.

11. Carvalho A, Rea IM, Parimon T, Cusack BJ. Physical activity and cognitive function in individuals over 60 years of age: a systematic review. Clin Interv Aging. 2014;9:661-82. Epub 2014/04/22. doi: 10.2147/cia.S55520. PubMed PMID: 24748784; PubMed Central PMCID: PMCPMC3990369.

12. De Mello MT, de Aquino Lemos V, Antunes HKM, Bittencourt L, Santos-Silva R, Tufik S. Relationship between physical activity and depression and anxiety symptoms: a population study. Journal of affective disorders. 2013;149(1-3):241-6.

13. Teixeira CM, Vasconcelos-Raposo J, Fernandes HM, Brustad RJ. Physical activity, depression and anxiety among the elderly. Social Indicators Research. 2013;113(1):307-18.

14. Zhao Y, Hu Y, Smith JP, Strauss J, Yang G. Cohort profile: the China health and retirement longitudinal study (CHARLS). International journal of epidemiology. 2014;43(1):61-8.

15. Zhou X, Liao S, Qi L, Wang R. Physical activity and its association with cognitive function in middle-and older-aged Chinese: Evidence from China Health and Retirement Longitudinal Study, 2015. European Journal of Sport Science. 2021:1-11.

16. Yang R, Xu D, Wang H, Xu J. Longitudinal trajectories of physical functioning among Chinese older adults: the role of depressive symptoms, cognitive functioning and subjective memory. Age and Ageing. 2021;50(5):1682-91.

17. Boey KW. Cross-validation of a short form of the CES-D in Chinese elderly. International journal of geriatric psychiatry. 1999;14(8):608-17. 
18. Van Buuren S, Groothuis-Oudshoorn K. mice: Multivariate imputation by chained equations in R. Journal of statistical software. 2011;45:1-67.

19. Zaninotto P, Batty GD, Allerhand M, Deary IJ. Cognitive function trajectories and their determinants in older people: 8 years of follow-up in the English Longitudinal Study of Ageing. J Epidemiol Community Health. 2018;72(8):685-94.

20. Murphy RA, Hagaman AK, Reinders I, Steeves JA, Newman AB, Rubin SM, et al. Depressive trajectories and risk of disability and mortality in older adults: longitudinal findings from the health, aging, and body composition study. Journals of Gerontology Series A: Biomedical Sciences and Medical Sciences. 2016;71(2):228-35.

21. Angevaren M, Vanhees L, Wendel-Vos W, Verhaar HJ, Aufdemkampe G, Aleman A, et al. Intensity, but not duration, of physical activities is related to cognitive function. European Journal of Preventive Cardiology. 2007;14(6):825-30.

22. Dietrich A, Audiffren M. The reticular-activating hypofrontality (RAH) model of acute exercise. Neuroscience \& Biobehavioral Reviews. 2011;35(6):1305-25.

23. Ge L, Yap CW, Ong R, Heng BH. Social isolation, loneliness and their relationships with depressive symptoms: a population-based study. PloS one. 2017;12(8):e0182145.

24. Loprinzi PD. Objectively measured light and moderate-to-vigorous physical activity is associated with lower depression levels among older US adults. Aging \& mental health. 2013;17(7):801-5.

25. Kandola A, Lewis G, Osborn DP, Stubbs B, Hayes JF. Depressive symptoms and objectively measured physical activity and sedentary behaviour throughout adolescence: a prospective cohort study. The Lancet Psychiatry. 2020;7(3):262-71.

26. WHO. Physical activity 2020 [updated 26 Nov, 2020]. Available from: https://www.who.int/news-room/fact-sheets/detail/physical-activity.

\section{Tables}

Table 1 Characteristic of subjects

\begin{tabular}{|c|c|c|c|c|}
\hline Variables & Wave 1 & Wave 2 & Wave 3 & Wave 4 \\
\hline & Mean(SD)/N(\%) & Mean(SD)/N(\%) & Mean(SD)/N(\%) & Mean(SD)/N(\%) \\
\hline \multicolumn{5}{|l|}{ Physical Activity Intensity(days/week) } \\
\hline Vigorous physical activity a,b & $0(5)$ & $0(4)$ & $0(4)$ & $0(2)$ \\
\hline Moderate physical activity ${ }^{a, b}$ & $4(7)$ & $2(7)$ & $2(7)$ & $0(7)$ \\
\hline Light physical activity $\quad a, b$ & $7(2)$ & $7(4)$ & $7(4)$ & $7(4)$ \\
\hline Cognitive function $(0-30)^{a}$ & $14.9(4.7)$ & $15.6(4.5)$ & $14.9(4.6)$ & $15.6(5.2)$ \\
\hline Depression (CES-D score,0-30) & $7.9(6.1)$ & $7.6(5.6)$ & $7.7(6.2)$ & $8.6(6.5)$ \\
\hline Age (years) & $57(13)$ & & & \\
\hline Sex (Female) & $2545(49.4 \%)$ & & & \\
\hline \multicolumn{5}{|l|}{ Educational attainment } \\
\hline No formal education & 1972(39.2\%) & & & \\
\hline Elementary school and middle school & $2316(46.1 \%)$ & & & \\
\hline High school and higher & $737(14.7 \%)$ & & & \\
\hline \multicolumn{5}{|l|}{ Marital status } \\
\hline Married/partnered & $4479(89.1 \%)$ & & & \\
\hline Unmarried & $546(10.9 \%)$ & & & \\
\hline Residence location (Rural) & $2860(56.9 \%)$ & & & \\
\hline Co-residence with children & $2933(58.4 \%)$ & & & \\
\hline Number of chronic diseases & $1(2)$ & & & \\
\hline $\operatorname{ADLs}(0-6)$ & $0(0)$ & & & \\
\hline Subjective memory (1-5) & $4.0(0.9)$ & & & \\
\hline
\end{tabular}

Note: Unmarried includes separated/divorced/widowed/never married.

a Vigorous physical activity: wave $1 \mathrm{n}=5009$; wave $2 \mathrm{n}=3689$; wave $3 \mathrm{n}=2106$; wave $4 \mathrm{n}=3999$; 
Moderate physical activity: wave $1 \mathrm{n}=5008$; wave $2 \mathrm{n}=3689$; wave $3 \mathrm{n}=2098$; wave $4 \mathrm{n}=3999$;

Light physical activity: wave $1 \mathrm{n}=4995$; wave $2 \mathrm{n}=3675$; wave $3 \mathrm{n}=2104$; wave $4 \mathrm{n=3999}$;

Cognitive function: wave $1 n=5025$; wave $2 n=3617$; wave $3 n=3606$; wave $4 n=2672$;

Depression: wave $1 \mathrm{n}=5025$; wave $2 \mathrm{n}=4198$; wave 3=4125; wave 4=3764;

${ }^{\mathrm{b}}$ Median and Interquartile range were presented in these variables: vigorous physical activity, moderate physical activity, light physical activity, ADLs, and number of chronic diseases.

Table 2 The impact of physical activity intensity on the trajectories of cognitive function and depressive symptoms

\begin{tabular}{|c|c|c|c|c|c|c|}
\hline & \multicolumn{3}{|c|}{ Cognitive Function } & \multicolumn{3}{|c|}{ Depressive Symptoms } \\
\hline & $\beta$ & SE & $95 \% \mathrm{Cl}$ & $\beta$ & SE & $95 \% \mathrm{Cl}$ \\
\hline Wave & -0.24 *夫 & 0.08 & {$[-0.39,-0.10]$} & $0.46^{\star \star \star}$ & 0.09 & {$[0.28,0.64]$} \\
\hline Vigorous PA & $-0.06^{\star}$ & 0.03 & {$[-0.10,0.01]$} & 0.05 & 0.04 & {$[-0.02,0.13]$} \\
\hline Moderate PA & -0.01 & 0.03 & {$[-0.06,0.04]$} & 0.04 & 0.03 & {$[-0.03,0.10]$} \\
\hline Light PA & 0.02 & 0.03 & {$[-0.05,0.07]$} & 0.06 & 0.04 & {$[-0.02,0.13]$} \\
\hline Wave*vigorous PA & -0.01 & 0.01 & {$[-0.03,0.01]$} & 0.01 & 0.01 & {$[-0.02,0.04]$} \\
\hline Wave*moderate PA & $0.02^{\star}$ & 0.01 & {$[0.002,0.04]$} & -0.02 & 0.01 & {$[-0.05,0.003]$} \\
\hline Wave*light PA & 0.02 & 0.01 & {$[-0.01,0.04]$} & $-0.03^{*}$ & 0.01 & {$[-0.06,-0.01]$} \\
\hline Age & $-0.10 * \star \star$ & 0.01 & {$[-0.11,-0.09]$} & $-0.03 \star \star \star$ & 0.01 & {$[-0.04,-0.02]$} \\
\hline Sex & $-0.16^{*}$ & 0.08 & {$[-0.32,0.00]$} & $1.25^{\star \star \star}$ & 0.10 & {$[1.04,1.46]$} \\
\hline \multicolumn{7}{|c|}{ Educational attainment (ref: no formal education) } \\
\hline Elementary school and middle school & 2.93 & 0.09 & {$[2.76,3.10]$} & $-0.85^{\star \star \star}$ & 0.11 & {$[-1.07,-0.63]$} \\
\hline High school and higher & 4.58 & 0.13 & {$[4.34,4.83]$} & $-1.72^{\star \star \star}$ & 0.16 & {$[-2.04,-1.40]$} \\
\hline Marital status(ref:married) & 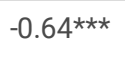 & 0.14 & {$[-0.91,-0.37]$} & $0.95^{\star \star \star}$ & 0.17 & {$[0.61,1.28]$} \\
\hline Residence location(ref: urban) & 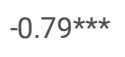 & 0.08 & {$[-0.95,-0.63]$} & $0.95^{\star \star \star}$ & 0.11 & {$[0.75,1.16]$} \\
\hline Co-residence with children(ref:No) & $-0.29 \star \star \star *$ & 0.08 & {$[-0.45,-0.13]$} & 0.004 & 0.10 & {$[-0.20,0.21]$} \\
\hline Number of chronic diseases & 0.05 & 0.03 & {$[-0.01,0.11]$} & $0.84 * \star \star$ & 0.04 & {$[0.77,0.91]$} \\
\hline $\operatorname{ADLs}(0-6)$ & 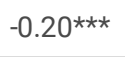 & 0.05 & {$[-0.30,-0.10]$} & 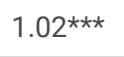 & 0.06 & {$[0.90,1.15]$} \\
\hline Subjective memory (1-5) & $-0.76^{\star \star \star}$ & 0.05 & {$[-0.85,-0.67]$} & $1.13^{\star \star \star}$ & 0.06 & {$[1.01,1.24]$} \\
\hline
\end{tabular}

Note: PA refers to physical activity; ADLs refer to activities of daily living.

*P $<0.05 ; * \star P<0.01 ; * \star * p<0.001$

\section{Figures}




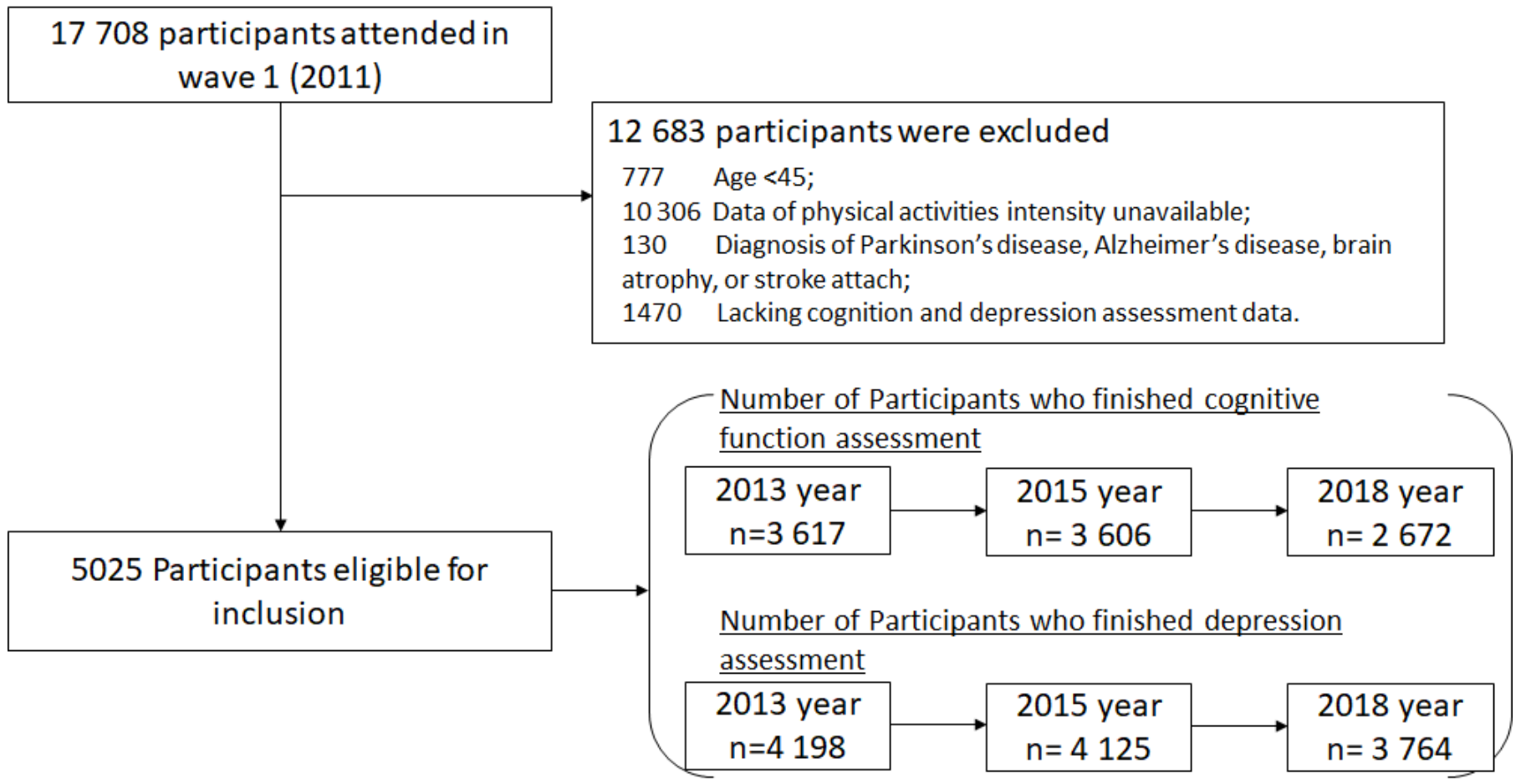

Figure 1

Flow chart showing the selection of the participants in this study

Cognitive function

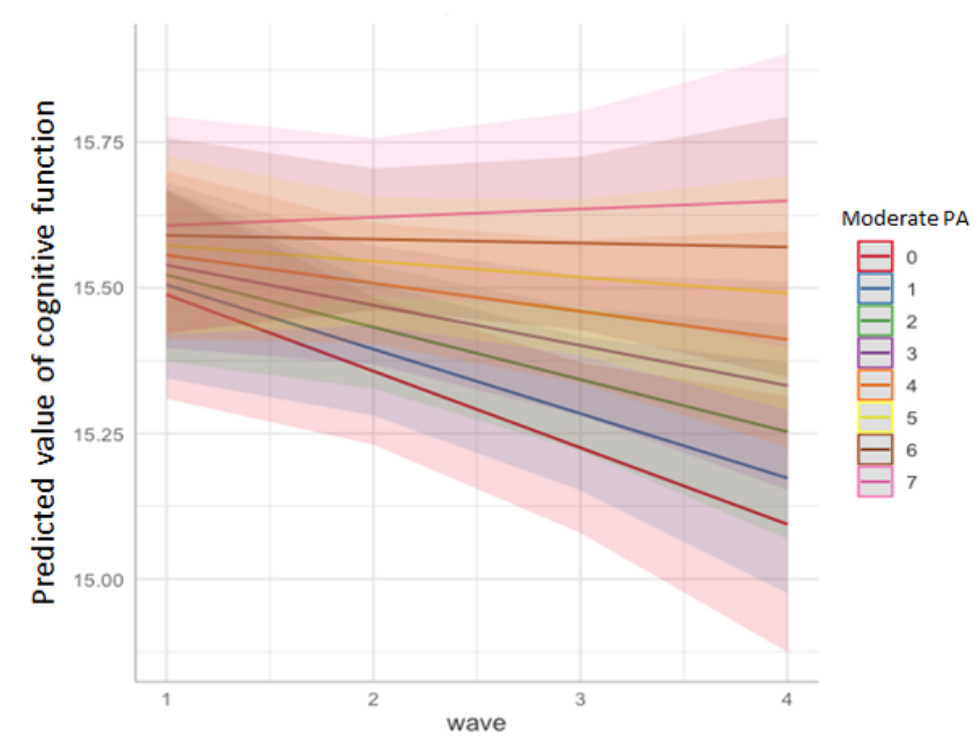

Depressive symptoms

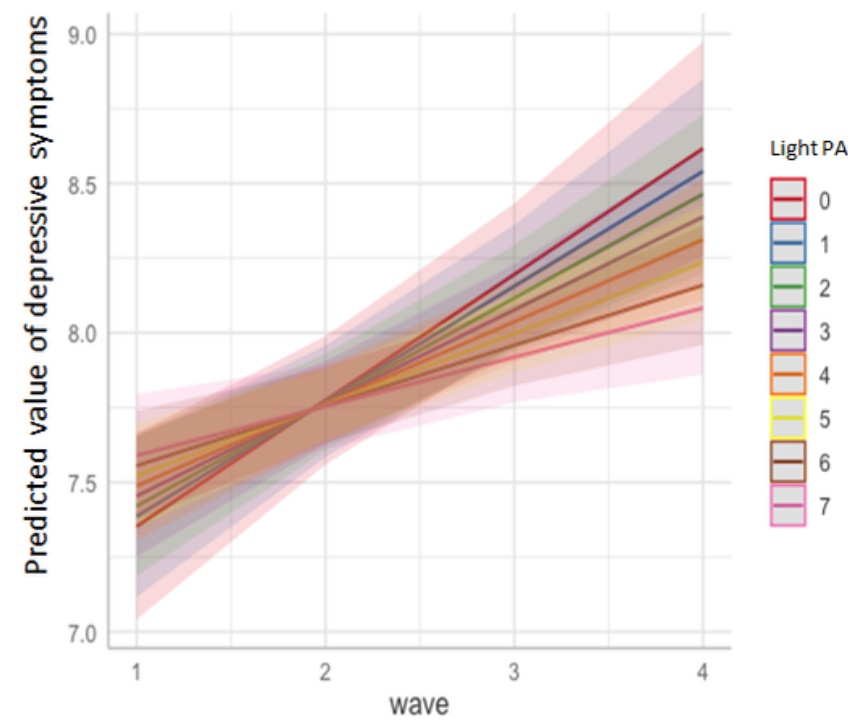

Figure 2

Estimated trajectories of cognitive function(left) and depressive symptoms(right) by moderated PA and light PA over time. Note: PA, physical activity.

\section{Supplementary Files}

This is a list of supplementary files associated with this preprint. Click to download.

- Supplementary.docx 